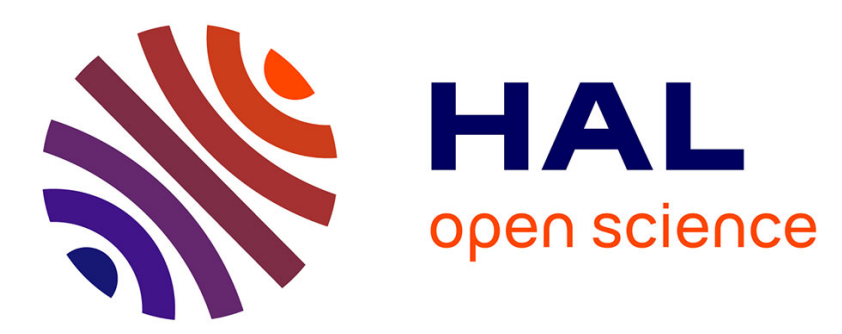

\title{
Description d'un piège à succion: son emploi dans la recherche des aphides vecteurs de virus transmis sur le mode non persistant
}

Gérard Labonne, Claude Fauvel, François Leclant, Jean-Bernard Quiot

\section{To cite this version:}

Gérard Labonne, Claude Fauvel, François Leclant, Jean-Bernard Quiot. Description d'un piège à succion: son emploi dans la recherche des aphides vecteurs de virus transmis sur le mode non persistant. Agronomie, 1982, 2 (8), pp.773-776. 10.1051/agro:19820812 . hal-02728842

\section{HAL Id: hal-02728842 \\ https://hal.inrae.fr/hal-02728842}

Submitted on 2 Jun 2020

HAL is a multi-disciplinary open access archive for the deposit and dissemination of scientific research documents, whether they are published or not. The documents may come from teaching and research institutions in France or abroad, or from public or private research centers.
L'archive ouverte pluridisciplinaire HAL, est destinée au dépôt et à la diffusion de documents scientifiques de niveau recherche, publiés ou non, émanant des établissements d'enseignement et de recherche français ou étrangers, des laboratoires publics ou privés. 


\title{
Description d'un piège à succion : son emploi dans la recherche des aphides vecteurs de virus transmis sur le mode non persistant
}

\author{
Gérard LABONNE, Christian FAUVEL $\left({ }^{*}\right)$, François LECLANT $\left({ }^{* *}\right)$ \& Jean-Bernard QUIOT \\ I.N.R.A., Station de Pathologie végétale, Centre de Recherches Antilles-Guyane, 97170 Petit-Bourg, \\ Guadeloupe. \\ (*) I.N.R.A., Station de Pathologie végétale, Centre de Recherches d'Avignon, F84140 Montfavet. \\ (**) E.N.S.A.M., Chaire de Zoologie, F 34060 Montpellier Cédex.
}

\section{RÉSUMÉ \\ Piège à succion, Piège de Moericke, Aphides, Vecteurs, Virus.}

\begin{abstract}
Le piège à succion décrit dans cette note a été réalisé afin de permettre le contrôle de la proportion de pucerons vecteurs d'un virus au niveau des feuilles de plantes maraîchères. Il est caractérisé par la faible hauteur du cône collecteur d'insectes ( $22 \mathrm{~cm}$ au-dessus du sol), par son rendement (sa puissance d'aspiration permet de capturer plus de 3 fois plus de pucerons qu'un piège jaune de Moericke de $30 \mathrm{~cm}$ de diamètre placé dans les mêmes conditions) et par la possibilité de recueillir les insectes vivants. Un essai utilisant Aphis gossypii a montré que le pourcentage de transmission du virus de la mosaïque du concombre n'était pas affecté par un séjour de $15 \mathrm{mn}$ des pucerons infectieux dans le piège.
\end{abstract}

\section{SUMMARY}

\section{Suction trap,}

Moericke trap, Aphids,

Vectors,

Virus.

\section{Description of a suction trap and its use for the monitoring of aphid vectors of non-persistent viruses}

The suction trap here described was designed for the monitoring of infectivity in virus-transmitting aphids. Its main features are low height above the ground of its collector $(22 \mathrm{~cm})$, its power (over 3 times as many aphids are trapped as in a $30 \mathrm{~cm}$ diameter Moericke yellow trap) and the fact that it catches the insects alive. In a test, alates of Aphis gossypii were not affected by a $15 \mathrm{mn}$ period in the suction trap in their capacity to transmit cucumber mosaic virus to young muskmelons.

\section{INTRODUCTION}

Les expérimentations en conditions naturelles qui nécessitent la connaissance de la faune entomologique du lieu se heurtent toujours au problème de l'échantillonnage des populations d'insectes présents. Tel est le cas notamment des observations épidémiologiques sur les maladies virales, pour lesquelles l'estimation des populations de vecteurs assurant la dissémination du virus est indispensable. Lorsque les vecteurs sont aériens, l'utilisation de pièges à succion tels que ceux décrits par JOHNSON \& TAYLOR (1955) permet de résoudre le problème: SouTHWOOD (1978) présente une synthèse des publications qui portent sur les conditions d'emploi et l'interprétation des données fournies par ces appareils.

Le nouveau type de piège à succion décrit dans cette note permet la capture d'insectes évoluant au niveau d'une végétation basse ou rampante. Son rendement est comparé à celui du piège coloré de Moericke placé dans les mêmes conditions. Son utilisation en vue de détecter, parmi les insectes capturés, ceux qui sont susceptibles de transmettre immédiatement un virus non-persistant est discutée.

\section{DESCRIPTION DU PIÈGE À SUCCION}

Le piège (fig. 1) est composé de 3 parties : une caisse, un extracteur d'air et un système de collecte des insectes.

La caisse du piège ainsi que les supports du ventilateur et du système de collecte des insectes sont en chlorure de polyvinyle de $3 \mathrm{~mm}$ d'épaisseur. Le collecteur d'insectes (fig. 2) est un cône en grillage de laiton (15 fils par $\mathrm{cm}$ ) dont l'extrémité inférieure est munie d'un système de 3 plaques permettant d'ajuster un récipient de récolte. Le tout se retire entièrement du bâti de façon à atteindre le récipient de récolte.

A la mise en place du piège, la caisse est enterrée de $25 \mathrm{~cm}$, de façon à assurer une continuité entre sa surface plane et le sol ; une manche à air en plastique transparent est montée sur la virole du ventilateur afin d'expulser l'air en dehors de la zone de prélèvement.

Ainsi réalisé, ce piège à succion permet d'effectuer des prélèvements à une hauteur de $22 \mathrm{~cm}$ au-dessus du sol en échantillonnant $3000 \mathrm{~m}^{3} / \mathrm{h}$ d'air par vent nul.

Plusieurs modifications peuvent être apportées, suivant le but recherché : 


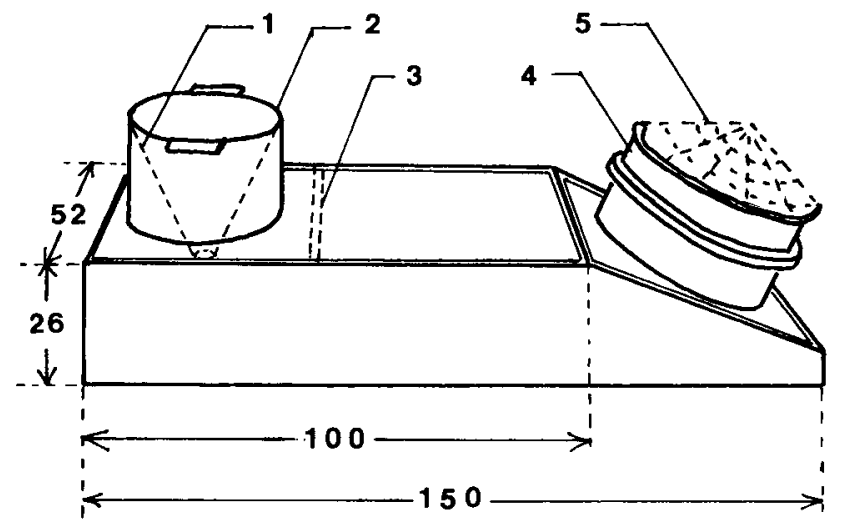

Figure 1

Piège à succion.

Suction trap.

(Les dimensions indiquées sont en $\mathrm{cm}$ ).

(Dimensions are set in $\mathrm{cm}$ ).

$1=$ Collecteur d'insectes. Insects collector.

2 = Support du collecteur $(30 \mathrm{~cm}$ de diamètre, $22 \mathrm{~cm}$ de haut $)$. Mount of the insects collector (diameter: $30 \mathrm{~cm}$; height: $22 \mathrm{~cm}$ ).

$3=$ Barre interne de rigidité. Internal bar for rigidity.

$4=$ Ventilateur (Diamètre intérieur: $40 \mathrm{~cm} ; 1400$ tours/mn). Fan (internal diameter: $40 \mathrm{~cm}$; speed : 1400 rotations $/ \mathrm{mn}$ ).

$5=$ Grillage de protection. Protecting wire-netting.

- la hauteur du support du collecteur d'insectes peut être supérieure à $22 \mathrm{~cm}$, de manière à s'adapter à la hauteur de la végétation environnante.

- un collecteur de fractions peut être adapté, permettant des récoltes à intervalles réguliers.

- lorsque l'appareil est en fonctionnement continu, un toit le protégeant de la pluie doit être réalisé. Dans notre cas, ce toit est constitué de 2 plaques de matière plastique transparente (onduline) à $1,70 \mathrm{~m}$ de haut de manière à perturber le moins possible le comportement de vol des insectes.

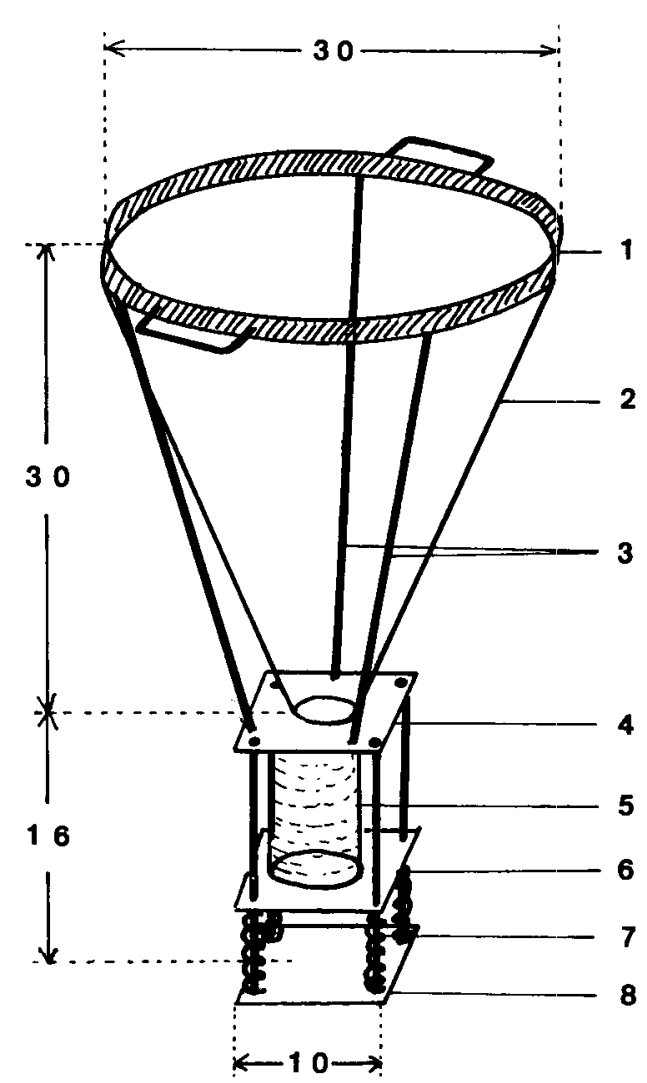

Figure 2

Collecteur d'insectes.

Insects collector.

(Les dimensions indiquées sont en $\mathrm{cm}$ ).

(Dimensions are set in $\mathrm{cm}$ ).

$1=$ Cercle support du cône. Mount of the cone.

2 = Cône en grillage de laiton. Copper wire-gauze cone.

$3=$ Barres de rigidité du cône. Bars for rigidity of the cone.

$4=$ Plaque fixe. Fixed plate.

$5=$ Flacon collecteur. Collecting bottle.

$6=$ Plaque mobile. Movable plate.

$7=$ Ressort. Spring.

$8=$ Plaque fixe. Fixed plate.

\section{TABLEAU 1}

Comparaison de 2 techniques de capture sur une période de $10 \mathrm{j}$ pour l'ensemble des Aphides et pour le groupe Aphis fabae Scop. Comparison of 2 trapping methods during a ten-day period. Results for the whole aphid population and for the group Aphis fabae Scop.

\begin{tabular}{|c|c|c|c|c|c|c|}
\hline \multirow{2}{*}{ Date } & \multicolumn{2}{|c|}{ Vitesse moyenne du vent $\left({ }^{*}\right)$} & \multicolumn{2}{|c|}{$\begin{array}{c}\text { Aphides, } \\
\text { toutes espèces confondues }\end{array}$} & \multicolumn{2}{|c|}{ Aphis groupe fabae } \\
\hline & à $2 \mathrm{~m}$ & à $0,2 \mathrm{~m}$ & PS & PM & PS & PM \\
\hline 13.VI.78 & 0,7 & 0,6 & 742 & 283 & 91 & 53 \\
\hline 14. VI. 78 & 0,4 & 0,3 & 753 & 264 & 64 & 31 \\
\hline 15.VI.78 & 0,5 & 0,4 & 709 & 256 & 72 & 35 \\
\hline 16.VI. 78 & 0,7 & 0,5 & 467 & 229 & 79 & 68 \\
\hline 17.VI.78 & 0,8 & 0,6 & 757 & 284 & 105 & 56 \\
\hline 18.VI. 78 & 0,8 & 0,6 & 1464 & 407 & 162 & 86 \\
\hline 19.VI. 78 & 0,8 & 0,6 & 1123 & 283 & 159 & 40 \\
\hline 20. VI. 78 & 0,5 & 0,4 & 756 & 180 & 110 & 23 \\
\hline 21.VI. 78 & 0,7 & 0,5 & 799 & 132 & 151 & 18 \\
\hline 22. VI. 78 & 1,5 & 0,7 & 786 & 130 & 106 & 18 \\
\hline Total & & & 8356 & 2448 & 1099 & 430 \\
\hline
\end{tabular}

PS = Piège à succion ; PM = Piège de Moericke.

$\left(^{*}\right)$ Données en $\mathrm{m} \cdot \mathrm{s}^{-1}$, établies sur la période de fonctionnement des pièges $(7 \mathrm{~h}$ à $22 \mathrm{~h})$.

PS $=$ Suction trap; $\mathrm{PM}=$ Moericke yellow trap.

$\left(^{*}\right)$ Mean speed of wind in $\mathrm{m} \cdot \mathrm{s}^{-1}$ from 0700 to 2200 each day (time of use of trap). 


\section{COMPARAISON DES CAPTURES DE PUCERONS PAR PIËGE À SUCCION ET PIĖGE JAUNE DE MOERICKE}

Deux pièges à succion du type décrit ci-dessus ont été utilisés pendant 3 saisons d'essais dans une parcelle marâ̂chère située à Montfavet, dans la basse vallée du Rhône (QUIOT et al.., 1979) afin de caractériser les populations de pucerons vecteurs du virus de la mosaïque du concombre (CMV). Le dispositif comprenait en outre des pièges de Moericke constitués par des cuvettes de $30 \mathrm{~cm}$ de diamètre et $15 \mathrm{~cm}$ de hauteur, peintes intérieurement en jaune (équivalent au jaune «Bouton d'Or» Ripolin 514, décrit par BRUNEL \& LANGOUET, 1970), remplies d'eau et posées sur le sol.

Le tableau 1 présente les captures sur une période de 10 jours, effectuées à l'aide d'un piège à succion et d'un piège de Moericke, situés à $6 \mathrm{~m}$ l'un de l'autre dans des conditions identiques (terrain nu au voisinage d'une parcelle de melon). Les espèces du groupe Aphis fabae Scop. ont été retenues afin d'exclure de la comparaison les espèces très peu attirées par le jaune (telle Hyalopterus pruni Geof., MOERICKE, 1969) ou au contraire très attirées par cette couleur.

\section{UTILISATION DU PIÈGE À SUCCION POUR DÉTECTER LA PRÉSENCE DE PUCERONS CAPABLES DE TRANSMETTRE LE VIRUS DE LA MOSAIIQUE DU CONCOMBRE (CMV)}

Les insectes capturés par le piège à succion peuvent être recueillis vivants : il suffit que le récipient de récolte soit vide et sec. Il est alors possible de contrôler les individus appartenant aux espèces supposées vecteurs de virus afin de détecter la présence éventuelle du (ou des) virus recherché(s). Pour cela les insectes sont prélevés avec un microaspirateur dans le récipient de récolte puis déposés sur des plantes saines, sensibles au virus, sur lesquelles l'apparition des symptômes de virose est surveillée. Plumb (1976) et A'BRoOK \& DEWAR (1980) ont déjà employé cette méthode avec succès dans le cas d'un virus transmis sur le mode persistant : le virus de la jaunisse nanisante de l'orge en utilisant d'autres types de pièges à succion.

Cependant, le problème de la rétention du virus se pose lorsqu'il s'agit de détecter la présence d'un virus non persistant.

Deux éléments entrent alors en jeu :

1. le «stress» subi par l'insecte balayé par un courant d'air desséchant lorsqu'il est capturé ;

2. la période d'attente entre l'instant de la capture et celui où il peut piquer la plante sur laquelle il est déposé.

L'évolution du pouvoir infectieux d'un puceron en fonction de la période d'attente après l'acquisition du virus a été déterminée en conditions contrôlées dans le cas du virus de la mosaïque du concombre (B. DuFour, comm. pers.). Utilisant 2 de ses vecteurs, Aphis craccivora Koch et Aphis gossypii Glov., cet auteur a montré que l'évolution est identique avec les 3 isolats de CMV utilisés, mais différente chez les 2 espèces : le pourcentage de transmission du virus au cours de la $1^{\text {re }}$ heure d'attente reste sensiblement identique avec $A$. gossypii tandis qu'il devient nul avec $A$. craccivora.

L'effet du «stress » subi par les pucerons capturés sur la rétention d'un virus non persistant a été analysé à partir d'un essai réalisé avec le couple CMV/A. gossypii. Dix adultes ailés d' $A$. gossypii ayant jeûné $1 \mathrm{~h}$ ont été placés pendant $3 \mathrm{mn}$ sur la plante source de virus, une petite cage les recouvrant. La source de virus est constituée par un melon (Cucumis melo L. var. "Védrantais ») inoculé $8 \mathrm{j}$ avant avec une souche locale de CMV (LEROUX et al., 1979). Pour chacune des 9 répétitions, les pucerons ainsi traités ont été répartis en 2 lots : l'un a été mis en attente dans des boîtes de Petri durant $15 \mathrm{mn}$ (lot A); l'autre a été introduit dans le piège à succion en fonctionnement pendant $15 \mathrm{mn}$ (lot B). Toutes ces manipulations ont été faites à une température voisine de $30^{\circ} \mathrm{C}$ (température extérieure sous abri). Les pucerons des 2 lots ont ensuite été repris et placés sur des melons au stade $1^{\text {rc }}$ feuille à raison de 2 individus par plante, par série de 5 plantes. L'expression des symptômes de virose a ensuite été comparée pour les 45 plantes de chaque lot (tabl. 2): les différences observées entre les 2 lots ne sont pas significatives. Dans les conditions de l'essai, le piège à succion n'induit donc pas une baisse de pouvoir infectieux pour les pucerons qui y ont séjourné.

\section{TABLEAU 2}

Comparaison du nombre de plantes virosées après contamination par 2 lots d'adultes ailés de A. gossypii porteur du CMV (2 pucerons par plante).

Number of plants infected after introduction of two groups of CMV. infectious alate Aphis gossypii. Two aphids were placed on each plant.

\begin{tabular}{|c|c|c|c|c|c|c|c|c|c|c|}
\hline $\begin{array}{l}\text { Répé- } \\
\text { tition }\end{array}$ & 1 & 2 & 3 & 4 & 5 & 6 & 7 & 8 & 9 & Total \\
\hline Lot $A$ & $2 / 5$ & $0 / 5$ & $1 / 5$ & $2 / 5$ & $3 / 5$ & $1 / 5$ & $1 / 5$ & $1 / 5$ & $1 / 5$ & $12 / 45$ \\
\hline Lot $B$ & $1 / 5$ & $1 / 5$ & $1 / 5$ & $1 / 5$ & $1 / 5$ & $1 / 5$ & $1 / 5$ & $1 / 5$ & $1 / 5$ & $10 / 45$ \\
\hline
\end{tabular}

Lot $\mathrm{A}=15 \mathrm{mn}$ d'attente en boîte de Petri.

Lot $\mathrm{B}=15 \mathrm{mn}$ à l'intérieur du piège à succion.

Numérateur $=\mathrm{nb}$ de plantes virosées $;$ Dénominateur $=\mathrm{nb}$ de plantes éprouvées.

Group $A=$ infectious aphids held for $15 \mathrm{mn}$ in a Petri dish. Group B $=$ infectious aphids held for $15 \mathrm{mn}$ in the suction trap. $\mathrm{x} / \mathrm{y}=$ number of infected plants/total number of plants.

\section{CONCLUSION}

Lc piège à succion décrit dans cette note permet l'échantillonnage des insectes en vol dans une strate située entre 20 et $30 \mathrm{~cm}$ au-dessus du sol. Utilisé pour recueillir les pucerons, il permet d'obtenir des individus nombreux et vivants pour lesquels il est possible de mettre en évidence s'ils sont ou non infectieux par un test biologique sur plante saine sensible au virus. Dans un essai portant sur $A$. gossypii et le virus de la mosaique du concombre, le séjour des pucerons dans le piège pendant $15 \mathrm{mn}$ n'a pas affecté la transmission du virus acquis au préalable. 


\section{RÉFÉRENCES BIBLIOGRAPHIQUES}

A'Brook J., Dewar A. M., 1980. Barley yellow dwarf virus infectivity of alate aphid vectors in West Wales. Ann. appl. Biol., 96, 5158.

Brunel E., Langouet L., 1970. Influence de caractéristiques optiques du milieu sur les adultes de Psila rosae Fab. (Diptères Psilidés): attractivité de surfaces colorées, rythme journalier d'activité. C.R. Soc. Biol., 164, 1638-1644.

Johnson C. G., Taylor L. R., 1955. The development of large suction traps for airborne insects. Ann. appl. Biol., 43, 51-62.

Leroux J. P., Quiot J. B., Lecoq H., Pitrat M., 1979. Mise en évidence et répartition dans le Sud-Est de la France d'un pathotype particulier du virus de la mosaïque du concombre. Ann. Phytopathol., 11, 431-438.
Moericke V.,1969. Host plant specific colour behaviour by Hyalopterus pruni (Aphididae). Entomol. exp. appl., 12, 524-534.

Plumb R. T., 1976. Barley yellow dwarf virus in aphids caught in suction traps. Ann. appl. Biol, 83, 53-59.

Quiot J. B., Marrou J., Labonne G., Verbrugghe M., 1979. Ecologie et épidémiologie du virus de la mosaïque du concombre dans le Sud-Est de la France. Description du dispositif expérimental. Ann. Phytopathol., 11, 265-282.

Southwood T.R.E., 1978. Ecological methods Chapman and Hall, London, $2^{\mathrm{c}}$ éd., $524 \mathrm{p}$. 